\title{
TRISOMÍA PARCIAL 4P POR CROMOSOMA 4 RECOMBINANTE ORIGINADO POR INVERSIÓN PERICÉNTRICA DE ORIGEN PATERNO
}

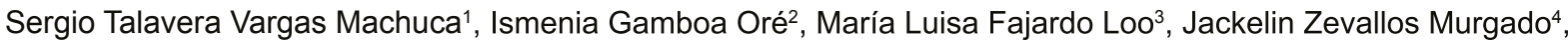 \\ Dina Torres Gonzales ${ }^{4}$, Liza Linares Rosales ${ }^{5}$, Leonor Contreras Aguilar ${ }^{5}$.
}

\begin{abstract}
RESUMEN
La trisomía parcial $4 p$, suele originarse de translocaciones entre el cromosoma 4 y otros cromosomas no homólogos o por inversión pericéntrica del cromosoma 4, originando gametos anómalos entre el cromosoma normal y el invertido, obteniéndose un cromosoma recombinante que puede portar duplicidad de segmentos del brazo pequeño (4p) o del brazo largo (4q) siendo este último inviable. Las características fenotípicas de esta condición cursan con retardo mental severo, retraso en el desarrollo psicomotor, anomalías craneofaciales, estrabismo, defectos osteomusculares, defectos cardiacos, criptorquidia y micropene. Describimos el caso de un niño de 1 año 6 meses con retardo en el desarrollo psicomotor y rasgos dismórficos, presentando una duplicación $4 p$ recombinante de origen paterno.Es el primer caso de duplicación $4 p$ hallado en nuestra institución y debido al carácter hereditario, es preciso realizar la consejería a los progenitores y seguimiento del propósitos para su manejo terapéutico y psicológico.
\end{abstract}

Palabras Clave: Trisomía 4p, Trisomía parcial 4p, Síndrome por duplicación $4 p$, Cromosoma 4 recombinante (Fuente: DeCS BIREME).

\section{PARTIAL TRISOMY 4P RECOMBINANT CHROMOSOME 4 CAUSED BY PERICENTRIC INVERSION OF PATERNAL ORIGIN}

\begin{abstract}
The partial trisomy $4 \mathrm{p}$, usually originated from translocations between chromosome 4 and other non-homologous chromosomes or pericentric inversion of chromosome 4, causing abnormal chromosome gametes between normal and the inverted one, yielding a recombinant chromosome duplication can carry small arm segments $(4 p)$ or the long arm (4q) the latter being non-viable. The phenotypic characteristics of this condition present with severe mental retardation, delayed psychomotor development, craniofacial anomalies, strabismus, musculoskeletal defects, heart defects, cryptorchidism and micropenis. We describe the case of a 1-year 6-month child with delay in psychomotor development and dysmorphic features, having a recombinant $4 p$ duplication of paternal origin. It is the first case of duplication $4 p$ found in our institution due to hereditary, it is necessary to perform counseling to parents and monitoring of the proband for therapeutic and psychological management.
\end{abstract}

Key words: Trisomy 4p; Partial trisomy 4p; $4 p$ duplication syndrome; Chromosome 4 recombinant (Source: MeSH NLM).

\section{INTRODUCCIÓN}

Las anomalías cromosómicas estructurales son menos frecuentes que las numéricas, de las cuales muchas, como las trisomías autosómicas 21,18 y 13 se encuentranbien caracterizadas. Una trisomía libre del cromosoma 4 es completamente inviable, pero una trisomía parcial del brazo corto del cromosoma 4 si es factible de hallar. Las duplicaciones cromosómicas de segmentos suelen provenir de portadores crípticos de reordenamientos cromosómicos balanceados, como es el caso de las inversiones pericéntricas. Debido a que los portadores no manifiestan ninguna evidencia fenotípica de estas anomalías cromosómicas, desconocen las probabilidades que tienen de ocurrencia o recurrencia para una descendencia afectada de un desbalance genómico. Una condición como la trisomía parcial $4 p$ es una afección incapacitante, que requiere un manejo terapéutico integral.

Las primeras descripciones de las características fenotípicas de los individuos afectados de trisomía parcial $4 p$ se remontan a cinco décadas atrás. Descripciones de Gustavson ${ }^{1}$,Wilson ${ }^{2}$ y posteriormente Schinzel ${ }^{3}$, detallan a pacientes con características fenotípicas peculiares donde se destacan un retardo en el crecimiento, dismorfias cráneo faciales, aplasia del hueso nasal, hendiduras palpebrales oblicuas, hipertelorismo, estrabismo, coloboma del iris dientes mal implantados, macroglosia, orejas grandes y de implantación baja, deformaciones de la columna vertebral, pie varo o zambo, talón prominente, hipospadias, criptorquidia y pene pequeño. Respecto

\footnotetext{
Biólogo. Docente de la Facultad de Medicina de la Universidad San Martín de Porres y de Ciencias de la Salud de la Universidad Norbert Wiener Laboratorio de Citogenética del Instituto Nacional Materno Perinatal. Lima-Perú.

2 Bióloga. Docente de la Facultad de Medicina de la Universidad Federico Villarreal y San Martín de Porres. Laboratorio de Citogenética del Instituto Nacional Materno Perinatal. Lima-Perú.

3 Médico Genetista. Instituto Nacional Materno Perinatal. Lima-Perú.

Bióloga. Laboratorio de Citogenética del Instituto Nacional Materno Perinatal. Lima-Perú.

Bachiller en Biología. Laboratorio de Citogenética del Instituto Nacional Materno Perinatal. Lima-Perú.
} 
a malformaciones de órganos, refieren cardiopatías, alteraciones del sistema osteomuscular y trastornos oculares. El retraso mental y un pobre desarrollo psicomotor así como los defectos del habla son notables entre los individuos afectados. Las características fenotípicas mencionadas y descritas en un individuo son sugerentes de anomalías de origen cromosómico, sin embargo resulta muy difícil, si no se dispone de los recursos tecnológicos de la citogenética moderna, llegar a identificar en anomalías cromosómicas estructurales desbalanceadas, el origen de los segmentos insertados o delecionados, por lo que se hace indispensable realizar el cariotipo a los progenitores para concluir los casos. En la actualidad se realizan estudios a nivel molecular ${ }^{4,5}$ utilizando técnicas de hibridación in situ por fluorescencia (FISH) y marcadores de secuencias específicos para determinar los puntos exactos de quiebre y las condiciones genéticas asociadas a las deleciones/duplicaciones. Estos estudios tienen gran relevancia, pues si se demuestra el compromiso hereditario de la afección, la consejería genética debe ir dirigida a la prevención y estimación de riesgos de recurrencia en los siguientes embarazos. Siendo este tipo de anomalía de carácter irreversible en los individuos afectados, el manejo clínico debe centrarse en mejorar la calidad de vida de los pacientes lo que implica un plan de atención multidisciplinario en donde participan diversas especialidades, como genética, cardiología, neurología, oftalmología, endocrinología, terapia física y rehabilitación. Para los progenitores, se requiere de apoyo psicológico que ayude a superar el impacto emocional que acarrea en el entorno familiar este tipo de afecciones.

\section{PRESENTACIÓN DEL CASO}

Se recibe en el consultorio de Genética a un niño de 1 año y seis meses de edad, de sexo masculino nacido a término por parto eutócico a las 38 semanas, en Hospital Nacional Docente Madre Niño San Bartolomé, sin control prenatal,peso al nacer $3145 \mathrm{~g}$ talla $49 \mathrm{~cm}$, circunferencia cefálica $34 \mathrm{~cm}$ y una puntuación de Apgar de 8 al minuto y 9 a los cinco minutos, la placenta fue normal y el líquido amniótico meconial color verde claro.Al examen físico evidencia caput succedaneum parietal derecho, macroglosia, paladar ojival, piel redundante en el cuello, protrusión a nivel epigástrico, micropene y fosa pilonidal a nivel de columna, frecuencia cardiaca 140 por minuto, frecuencia respiratoria 44 por minuto, grupo sanguíneo $O$ Rh (+), HIV (-), VDRL/RPR (-).Hijo de madre de 36 años multípara G6P6006 y padre de 28 años,con sospecha de síndrome genético por determinar, con retraso psicomotor, dismorfia, conducta autista e hiperactividad, sin manifestar movimientos involuntarios. Dentro de los antecedentes familiares se tiene abuela paterna y tíopaterno con diabetes mellitus, la abuela padece de hipertensión arterial. Al examen físico el propósitus presenta un peso de $11,35 \mathrm{~kg}$, talla $79 \mathrm{~cm}$, perímetro cefálico $46.5 \mathrm{~cm}$, facies atípica, dismorfismo craneofacial.
Piel con huellas de erosiones tipo mordedura en manos, el resto es normal. Se reconoce estrabismo, orejas dismórficas, aparente hipoacusia, dientes distróficos, en extremidades superiores, línea simiana unilateral. El tóraxes de tamaño y constitución normal, su frecuencia cardiaca es de 100 por min, revela soplo sistólico, las características del abdomen son normales. En genitales se observa criptorquidia bilateral y micropene. Al examen neuropediátrico evidencia un retraso global del desarrollo, con sospecha de síndrome de Lesch-Nyhan, En la evaluación del desarrollo psicomotriz se presentó hipotonía generalizada,no logra sentarse, se voltea, no transfiere objetos, ni fija la mirada en rostros, parece no escuchar, solo responde ante ruidos intensos, lenguaje mínimo, solo pronuncia la palabra mamá, muestra una conducta de autoagresión, mordiéndose las manos y golpeándose la cara. Evaluado por endocrinología se diagnostica trastorno de la diferenciación sexual, hipogonadismo, sospecha de síndrome de Hurler, se presume alguna condición de origen cromosómico. Por radiografía de columna lumbosacra se evidencia acentuación de cifosis fisiológica dorsal y discreta escoliosis dorso lumbar, el resto de estructuras óseas vertebrales de aspecto normal. La figura 1 ilustra algunas características fenotípicas del propositus.

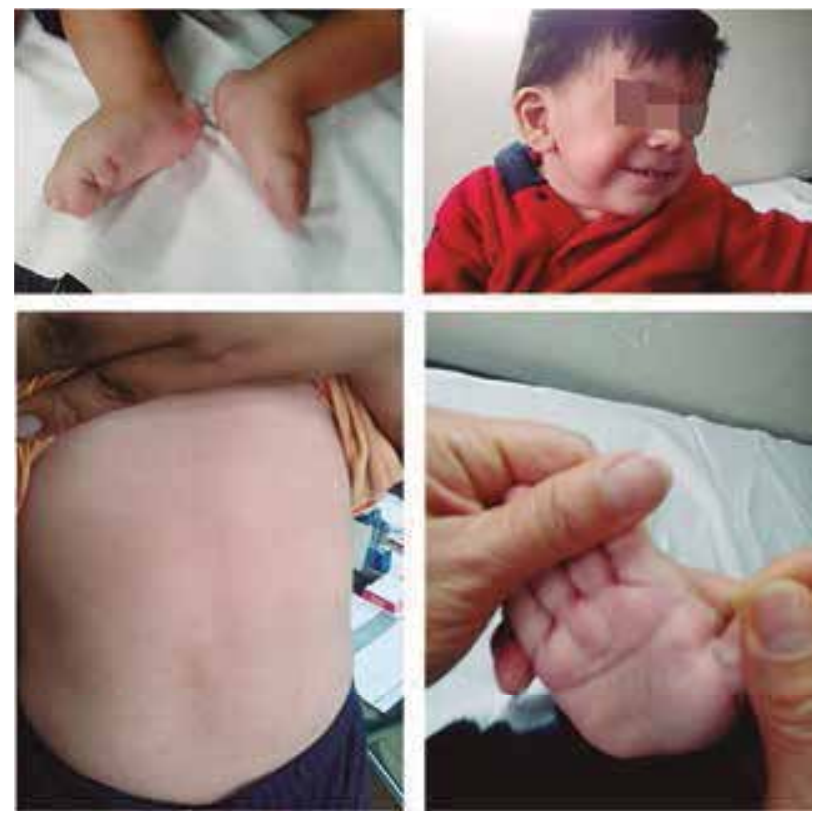

Figura 1. Aspectos fenotipicos del propositus. a. Pie en mecedora, obsérvese el talón prominente. b. Características dismórficas cráneo faciales. c. Cifosis pronunciada (flecha). d. Linea simiana derecha (flecha)

Al propositus se le realizó el cariotipo en sangre periférica (cultivo de linfocitos) en medio de cultivo RPMI 1640a $37^{\circ} \mathrm{C}$ durante 72 horas, luego de la cosecha, fueron preparadas las láminas para bandeo GTG (TripsinaGiemsa) realizando las lecturas a una resolución de 550 bandas con un microscopio Carl Zeiss Jenamed a 1000 $X$. Se halló una constitución cromosómica heterocigótica desbalanceada con un segmento duplicado $4 p$ en el brazo 


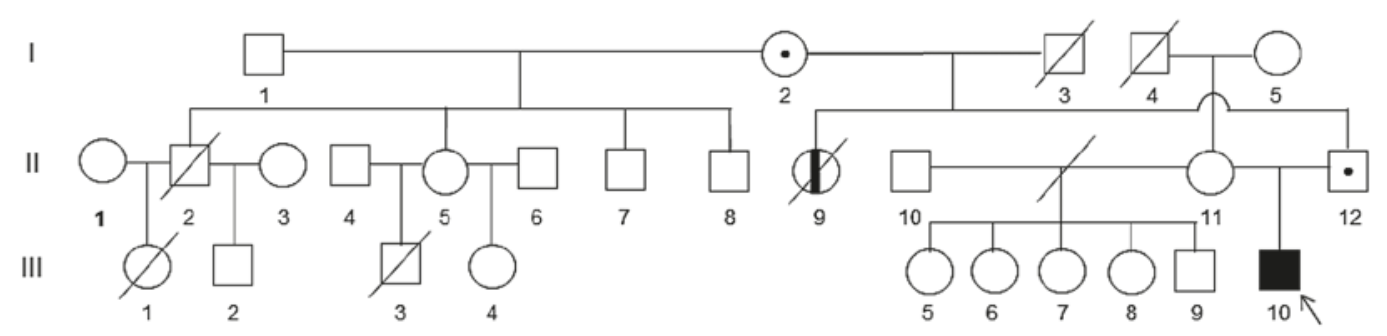

Figura 2. Árbol Genealógico Familiar mostrando la herencia de la condición a través de la línea paterna del propositus (flecha).

largo de uno de los cromosomas 4. Se realizó cariotipo a los progenitores del propositus encontrándose una alteración balanceada en el padre, mostrando una inversión pericéntrica en uno de los cromosomas $4(46, \mathrm{XY}, \operatorname{inv}(4)$ (p16.3q35.2)mat el cariotipo de la madre del propositus tuvo una constitución normal $(46, \mathrm{XX})$, el cariotipo del propósitos reveló un reordenamiento de tipo $46, \mathrm{XY}, \mathrm{rec}(4)$ dup(4p)inv(4)(p14q35.1)pat compatible con trisomía parcial $4 \mathrm{p}$, El estudio cromosómico se realizó mediante técnica citogenética clásica por método de bandeo GTG realizando las lecturas a una resolución de 500 bandascon un microscopio Carl Zeiss Jenamed a 1000X. Se encontró un patrón constante en 20 metafases analizadas en cada uno de los cariotipos. El árbol genealógico de la figura 2, nos muestra la herencia desde la línea paterna del propósitus con individuos, portadores, un afectado y otro probablemente afectado. Los cariogramas a partir de

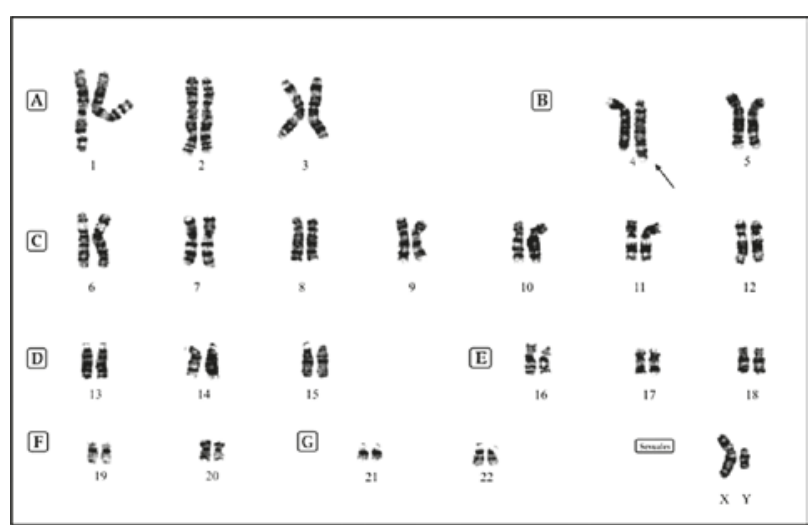

Figura 3. Cariograma del padre del propositus que muestra la inversión pericéntrica (flecha) en uno de los cromosomas 4.

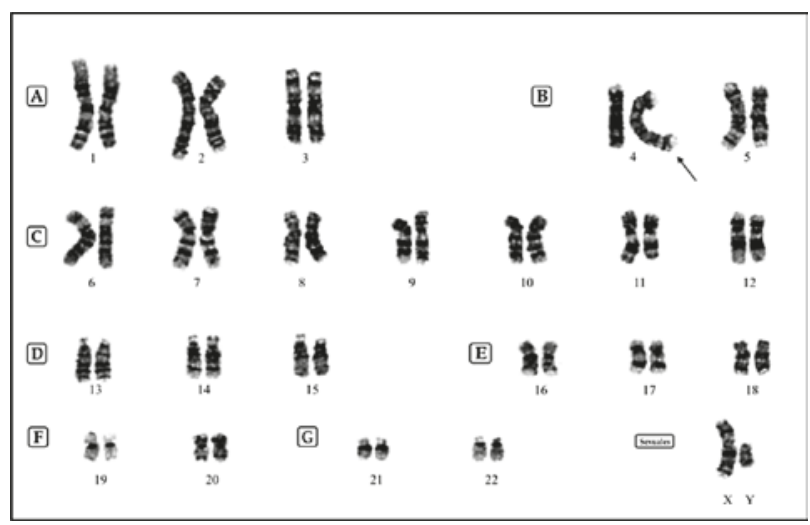

Figura 4. Cariograma del propositus que muestra la duplicación (flecha) del segmento $4 p$ en uno de los cromosomas 4 .

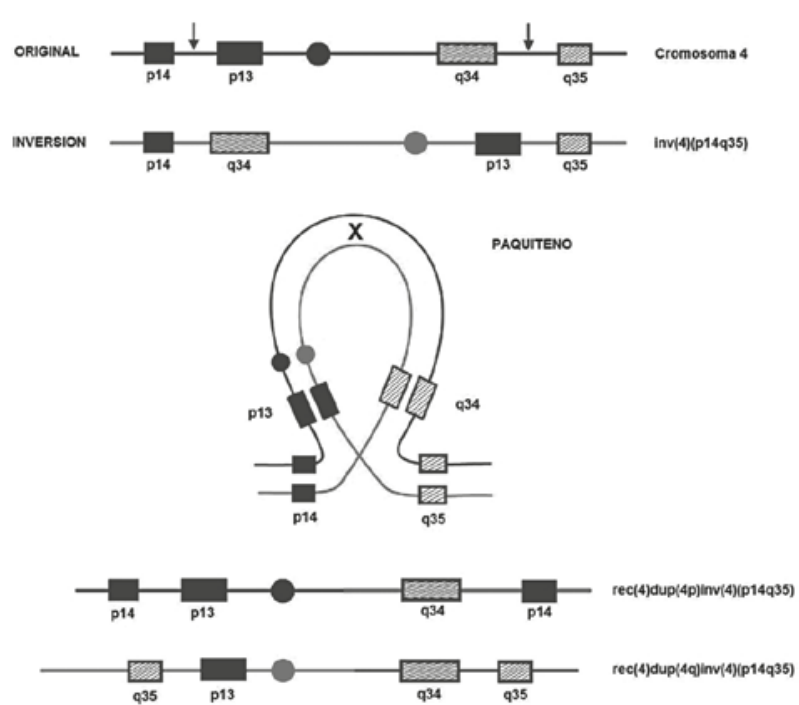

Figura 5. Mecanismo de recombinación entre el cromosoma 4 con inversión pericéntrica y su homólogo normal durante el paquiteno de la primera división meiótica, el resultado de esta recombinación anómala es el cromosoma recombinante 4 con duplicación de 4p (recuadro). Adaptado de ISCN 2009 An International System for Human Cytogenetic Nomenclature Karger AG Basel 2009.

los cuales se concluye la constitución cromosómica y su condición hereditaria del padre del propositus (figura 3) y la del propositus (figura 4), grafican los reordenamientos que involucran al cromosoma 4 o a uno de sus segmentos (4p). El mecanismo de origen (27) de este cromosoma recombinante así como una comparación entre un cromosoma 4 normal y los alterados son mostrados en las figura 5 y 6 respectivamente.

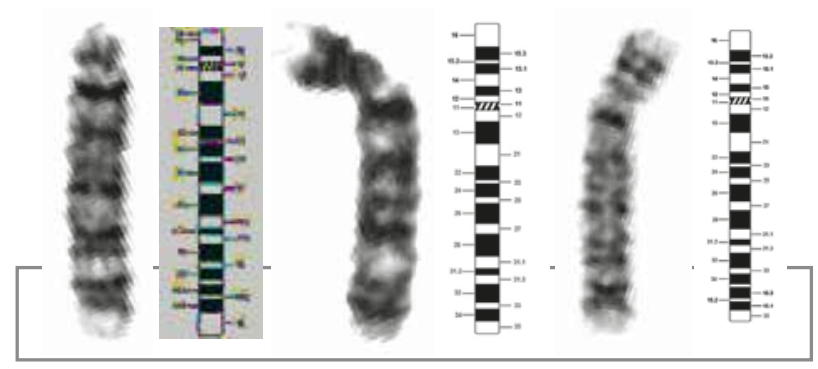

Figura 6. Comparación del cromosoma 4 normal (centro) con la inversión del progenitor masculino (izquierda) y la duplicación de $4 p$ en el cromosoma 4 del propósitus (derecha). 


\section{DISCUSIÓN}

Las evidencias fenotípicas de trisomía parcial $4 p$ encontradas en el propositus, son compatibles con aquellas descritas primero por diversos autores $1,2,3,6$, en nuestro caso, el paciente muestra un claro retraso en el desarrollo psicomotor, incapacidad para sentarse yla deambulación, marcada tendencia a la autoagresión y una conducta con rasgos autistas e incapacidad para fijar la mirada y déficit de atención. Estrabismo y maloclusión dentaria igualmente son rasgos del síndrome mencionados por De Grouchy ${ }^{7}$. Las anomalías de los genitales también han sido descritas ${ }^{3,}$ ${ }^{6}$, el propositus muestra criptorquidia y micropene, existe deformidades de las extremidades pies en mecedora con talón prominente y la deformación de la columna vertebral que también coincide con lo reportado ${ }^{3,6,8,9}$.

Esta condición genética usualmente se origina por translocaciones recíprocas que involucran el segmento $4 p$ con algún otro cromosoma no homólogo 1, 3, 6, 8, 10-13, sin embargo, puede aparecer tambiénesta anomalía por duplicaciones de segmentos del brazo corto del cromosoma 4 (4p), originado de inversiones pericéntricas del cromosoma 4, presente en alguno de los progenitores $2,4,5,12,14-18,25$, cuando esto sucede, nos referimos entonces a un cromosoma recombinante (rec). Demostramos mediante estudio citogenético a partir de cultivos de linfocitos de sangre periférica, la presencia de un cromosoma recombinante 4 en el propositus, con duplicación $4 p$ con fórmula cromosómica $46, X Y, r e c(4)$ dup(4p)inv(4)(p14q35.1)pat. Al realizar el cariotipo a los progenitores, la madre mostró un cariotipo normal $46, \mathrm{XX}$ y el padre una inversión pericéntrica de tipo $46, X Y$,inv(4) (p16.3q35.2)mat. Obtener la conclusión del estudio en el propósitus tuvo cierta dificultad, pues fue necesaria la repetición en dos oportunidades de la toma de muestra por falla en el crecimiento celular, lo que sugiere presencia de factores inhibitorios de respuesta a los mitógenos en la muestra de sangre, no obstante, se logró colectar suficiente evidencia en las lecturas efectuadas.

Hasta el año 2002 Battaglia y cols reporta 85 casos de síndrome de duplicación $4 p$ (trisomía $4 p$ ) principalmente debido a translocaciones desbalanceadas, y 5 casos de síndrome recombinante 4 originado a partir de una inversión pericéntrica, con constitución cromosómica similar a nuestro caso ${ }^{18}$.

Es importante manifestar que el laboratorio no cuenta con recursos modernos para la identificación de los puntos de corte exactos en los segmentos cromosómicos implicados, pues compromete bandas claras en los extremos de ambos brazos del cromosoma 4. En la actualidad se utilizan pruebas citogenéticas moleculares para localizar los puntos de corte y reordenamiento aplicando sondas específicas para hibridación in situ por fluorescencia, o el uso de micro Arrays para Hibridación Genómica Comparativa(CGH)4, 5, 9, 16, 19, 24 . Los segmentos cromosómicos de $4 p$ involucrados se encuentran cerca de la región crítica para la deleción
$4 p$ - manifiesta en el Síndrome de Wolf Hirschhorn con características fenotípicas diferentes a la trisomía parcial $4 p$, reconocidas ya por pruebas moleculares $5,15,16,20$ debido a la imposibilidad de efectuar estas pruebas en nuestro medio no descartamos la posibilidad de esta condición en el propositus. El estudio citogenético del paciente mostró una línea celular pura lo que descarta mosaicismo para esta condición reportado en individuos fenotípicamente normales ${ }^{21}$. Es indispensable la evaluación y seguimiento por otras especialidades como terapia física y rehabilitación, cardiología, endocrinología y oftalmología ya que muchas de las características fenotípicas de la condición se harán evidentes con la edad ${ }^{12,20,22,26 .}$

Resulta notable la publicación de Eduardo Pasaró ${ }^{8}$ sobre los aspectos psicológicos y evolutivos conductuales en la rehabilitación de pacientes con trisomía parcial $4 p$ en cuanto a control postural, lenguaje, coordinación visomotora y sociabilidad, y su aplicación para la mejora de la calidad de vida del paciente.

Finalmente queremos destacar la relevancia de este reporte, ya que es el primero reportado en nuestra institución y hasta donde tenemos conocimiento, no existen antecedentes de casos similares en el país. La caracterización citogenética y el establecimiento del fenotipo con que cursa este síndrome es producto de un trabajo coordinado y multidisciplinario. Desde el punto de vista médico científico es un valioso aporte, pues se afina más el manejo clínico del paciente que es la prioridad inmediata, asimismo es imperativa la consejería o asesoramiento genético a los progenitores para la prevención de futuras gestaciones con alto riesgo de recurrencia de esta condición hereditaria o una alta tasa de pérdidas del embarazo ${ }^{23,28}$

\section{AGRADECIMIENTOS}

Agradecemos la colaboración en la elaboración de este trabajo por los aportes de las diferentes especialidades médicas a la Dra. Gloria Larrabure de Endocrinología, Dra. Pilar Medina, Neuropediatría; Dra. Katty Gárate, Radiología; Dra. Isolina Aburto Cardiología y Dr. Pablo Velásquez, Jefe del Departamento de Neonatología, todos ellos Médicos del Instituto Nacional Materno Perinatal quienes participaron en la evaluación del paciente y brindaron valiosa información.

\section{REFERENCIAS BIBLIOGRÁFICAS}

1. Gustavson, K.H., Finley, S.C., Jalling, B. (1964) A 4-5/2122 chromosomal translocation associated with multiple congenital anomalies. ActaPaediat. Scand. 53, 172-181.

2. Wilson, M.G., et al. (1970). Inherited pericentric inversion of chromosome $\mathrm{n}^{\circ}$ 4. American Journal Human Genetics. 22 679-689.

3. Schinzel A A, Schmid W: Partial trisomy for the short arm of chromosome 4 with translocation $4 p-, 18 q+$ in the father. Humangenetik 15:163-171, 1972 
4. Hemmat M, Hemmat O, Anguiano A, Boyar FZ, El Naggar M, Wang JC, Wang BT, Sahoo T, Owen R, Haddadin M. Genotype-phenotype analysis of recombinant chromosome 4 syndrome: an array-CGH study and literature review. Genotype-phenotype analysis of recombinant chromosome 4 syndrome: an array-CGH study and literature review. Mol Cytogenet. 2013 May 2;6(1):17.

5. Malvestiti F, Benedicenti F, De Toffol S, Chinetti S, Höller A, Grimi B, Fichtel G, Braghetto M, Agrati C, Bonaparte E, Maggi F, SimoniG,Grati FR. Recombinant chromosome 4 from a familial pericentric inversion: prenatal and adulthood wolfHirschhorn phenotypes. Case Rep Genet. 2013; 2013: 306098.

6. Giraud, F MatteiJ.F., MatteiM.G. AymeS., Bernard R.La trisomie 4p Humangenetik6. XI. 1975, Volume 30, Issue 2, pp 99-108

7. De Grouchy, J., Turleau C. Atlas de las Enfermedades Cromosómicas, Editorial Marín, S.A. 1978 Barcelona pp 4042

8. Pasaro E., Méndez J. y Peralbo M. U Aspectos Psicológicos y Evolutivos en un sujeto con trisomia parcial $4 \mathrm{p}$ Psicothema1992. Vol. 4, n² 2, pp. 355-367

9. Patel SV, Dagnew H, Parekh AJ, Koenig E, Conte RA, Macera MJ, Verma RS. Clinical manifestations of trisomy $4 p$ syndrome. Eur J Pediatr. 1995 Jun; 154(6):425-31.

10. Hustinx WJ, Gabreëls JM, Kirkels VG, Korten JJ, Scheres JM, Joosten EM, Rutten FJ. Trisomy $4 p$ in a family with $A$ $\mathrm{t}(4 ; 15)$. Ann Genet. 1975 Mar; 18(1):13-9.

11. Dallapiccola B, Giovanelli G, Forabosco A. The radiological pattern associated with the trisomy of the short arm of chromosome No 4 PediatrRadiol. 1975 Jan 24; 3(1):34-40.

12. Gonzalez CH, Sommer A, Meisner LF, Elejalde BR, Opitz JM The trisomy $4 p$ syndrome: case report and review Am J Med Genet. 1977; 1(2):137-56.

13. Oorthuys J W, Gerssen-SchoorlK B, De PaterJ M, and De FranceH FA third case of de novo partial trisomy 4 p.J MedGenet. May 1989; 26(5): 344-345.

14. Rethoré MO, Dutrillaux B, Job JC, et al. [Trisomy $4 p$ due to recombination aneusomy of an inversion 4 (p 14, q 35)]. Ann Genet 17:109, 1974 109-14

15. Hirsch B, Baldinger S Pericentric inversion of chromosome 4 giving rise to $\operatorname{dup}(4 p)$ and $\operatorname{dup}(4 q)$ recombinants within a single kindred. Am J Med Genet. 1993 Jan 1;45(1):5-8.
16. Dufke A, Eggermann K, Balg S, Stengel-Rutkowski S, Enders $\mathrm{H}$, Kaiser PA second case of inv (4) pat with both recombinants in the offspring: rec dup(4q) in a girl with Wolf-Hirschhorn syndrome and rec dup(4p). Cytogenet Cell Genet. 2000

17. Stembalska A, Laczmanska I, Schlade-Bartusiak K, Czemarmazowicz H, Murawski M, Sasiadek MRecombinant chromosome 4 resulting from a maternal pericentric inversion in two sisters presenting consistent dysmorphic features.Eur J Pediatr. 2007

18. Battaglia A, Brothman A, Carey J.Recombinant 4 syndrome due to an unbalanced pericentric inversion of chromosome 4 Am J Med Genet. 2002 Sep 15; 112(1):103-6.

19. Wyandt $\mathrm{HE}^{1}$, Milunsky J, Lerner T, Gusella JF, Hou A, MacDonald M, Adekunle S, Milunsky A. Characterization of a duplication in the terminal band of $4 p$ by molecular cytogenetics. Am J Med Genet. 1993 Apr 1; 46(1):72-6.

20. Lurie losif W, Samochvalov Vladimir A Trisomy $4 p$ and ocular defects Br J Ophthalmol 1994 78: 415-417

21. Bernardini L, Sinibaldi L, Ceccarini C, Novelli A, Dallapiccola B Reproductive history of a healthy woman with mosaic duplication of chromosome 4p PrenatDiagn. 2005 Apr; 25(4):283-5

22. Kleczkowska $A^{1}$, Fryns JP, van den Berghe $H$. Trisomy of the short arm of chromosome 4: the changing phenotype with age.Ann Genet. 1992;35(4):217-

23. Wolf G.C, Mao J, Izquierdo $\underline{L}$, and JoffeG Paternal pericentric inversion of chromosome 4 as a cause of recurrent pregnancy loss. J Med Genet. Feb 1994; 31(2): 153-155

24. FaganK., SoubjakiV., Donald P., Turner G., and PartingtonM Fine molecular mapping of the $4 \mathrm{p} 16.3$ aneuploidy syndromes in four translocation families J MedGenet. Jun 2000; 37(6): 449-451.

25. Garcia-Heras J, Martin J. A rec (4) dup $4 p$ inherited from a maternal inv(4)(p15q35): case report and review Am J Med Genet. 2002 May 1;109(3):226-30

26. García E., Chamizo B. Sánchez M, Jiménez M Alteraciones endocrinas en una niña con trisomía $4 p$ An Pediatr.2002;57:170-1 - Vol. 57 Núm.2

27. Shaffer L.G., Slovak M.L. ,Campbell L.J. ISCN An International System for Human Cytogenetic Nomenclature KARGER (2009)

28. Demirhan O., Özgünen F., Taştemir D. Clinical Manifestations of Partial Trisomy $4 p$ Balkan Journal of Medical Genetics. January 2011Volume 13, Issue 2, Pages 61-63. 